Obser vat i on of Dynami c I nt er act i ons bet ween Fundament al and Second- Har moni c Modes in a $\mathrm{Hi}$ gh- Power Sub- Ter ahertz Gyr ot ron Oper at i ng i n Regi mes of Sof $t$ and Hard Sel f-Exci tat i on

\begin{tabular}{|l|l|}
\hline 著者 & $\begin{array}{l}\text { Sai to Ter uo, Tat emat su Yoshi nor i, Yamaguchi } \\
\text { Yuusuke, I keuchi Shi nj i, Ogasawar a Shi nya, } \\
\text { Yamada Naoki, I keda Ry osuke, Ogawa I sam, } \\
\text { I dehar a Toshi taka }\end{array}$ \\
\hline $\begin{array}{l}\text { j our nal or } \\
\text { publ i cat i on ti t l e }\end{array}$ & Physi cal Revi ew Let t er s \\
\hline vol une & 109 \\
\hline page r ange & $155001-1-155001-5$ \\
\hline year & $2012-10$ \\
\hline URL & ht t p: //hdl . handl e. net /10098/8070 \\
\hline
\end{tabular}




\title{
Observation of Dynamic Interactions between Fundamental and Second-Harmonic Modes in a High-Power Sub-Terahertz Gyrotron Operating in Regimes of Soft and Hard Self-Excitation
}

\author{
Teruo Saito, ${ }^{1}$ Yoshinori Tatematsu, ${ }^{1}$ Yuusuke Yamaguchi, ${ }^{1}$ Shinji Ikeuchi, ${ }^{1}$ Shinya Ogasawara, ${ }^{2}$ Naoki Yamada, \\ Ryosuke Ikeda, ${ }^{1}$ Isamu Ogawa, ${ }^{1}$ and Toshitaka Idehara ${ }^{1}$ \\ ${ }^{1}$ Recearch Center for Development of Far-Infrared Region, University of Fukui, 3-9-1 Bunkyo, Fukui 910-8507, Japan \\ ${ }^{2}$ Department of Energy Engineering and Science, Nagoya University, Nagoya 464-8463, Japan \\ (Received 2 July 2012; published 9 October 2012)
}

\begin{abstract}
Dynamic mode interaction between fundamental and second-harmonic modes has been observed in high-power sub-terahertz gyrotrons [T. Notake et al., Phys. Rev. Lett. 103, 225002 (2009); T. Saito et al. Phys. Plasmas 19, 063106 (2012)]. Interaction takes place between a parasitic fundamental or firstharmonic $(\mathrm{FH})$ mode and an operating second-harmonic $(\mathrm{SH})$ mode, as well as among SH modes. In particular, nonlinear excitation of the parasitic $\mathrm{FH}$ mode in the hard self-excitation regime with assistance of a SH mode in the soft self-excitation regime was clearly observed. Moreover, both cases of stable twomode oscillation and oscillation of the FH mode only were observed. These observations and theoretical analyses of the dynamic behavior of the mode interaction verify the nonlinear hard self-excitation of the FH mode.
\end{abstract}

Mode interaction has been one of the major research subjects of gyrotrons [1,2]. Recently, the development of sub-terahertz and terahertz gyrotrons has intensified because many new applications of high-frequency gyrotrons are anticipated $[3,4]$. Since gyrotrons in the sub-terahertz and terahertz region usually operate at the secondharmonic $(\mathrm{SH})$ resonance, except for higher harmonic operations in a large orbit gyrotron [5], new studies have arisen on the interaction between $\mathrm{SH}$ modes and fundamental or first-harmonic (FH) modes [6,7]. However, important physics issues such as the nonlinear excitation of a parasitic FH mode in the hard self-excitation regime are not discussed.

This paper reports the first observation of the nonlinear hard self-excitation of a parasitic FH mode assisted by an operating $\mathrm{SH}$ mode in the soft self-excitation regime in high-power sub-terahertz gyrotrons. Moreover, both cases of stable two-mode oscillation and oscillation of the FH mode only were observed, which indicates strong coupling between the hard and soft self-excited modes. These observations and theoretical analyses verify these physical effects $[1,2]$. Multimode oscillations of FH modes in a high-power millimeter wave gyrotron were interpreted as nonlinear excitations [8]. However, hard self-excitation was not shown. Reduction of the start oscillation current of a FH mode was reported as mode cooperation $[9,10]$. However, the FH mode was still in the soft self-excitation regime. In the present experiment, the frequency detuning parameter $\Delta$ for the FH mode is larger than 0.6. Then, the FH mode is definitely in the hard self-excitation regime [11,12].

High-power single-mode SH oscillation is more difficult to induce than the single-mode $\mathrm{FH}$ oscillation, because of mode interaction with parasitic $\mathrm{FH}$ modes. The $\mathrm{SH}$ oscillation of hundreds of kilowatts was realized in the centimeter wavelength region [13]. In the sub-terahertz range, a $20 \mathrm{~kW}$ level $\mathrm{SH}$ oscillation was realized [14]. Very recently, a sub-terahertz SH oscillation approaching $100 \mathrm{~kW}$ has been demonstrated [15-17].

As the beam voltage $V_{k}$ or the beam current $I_{b}$ increases, a chance for mode interaction rises. Figure 1 plots the start oscillation currents of relevant modes including the operating $\mathrm{SH} \mathrm{TE} \mathrm{TE}_{17,2}$ mode and the parasitic $\mathrm{FH} \mathrm{TE}_{4,3}$ mode in the gyrotron studied in Ref. [17]. The resonance frequency of the $\mathrm{TE}_{17,2}$ mode is about $390 \mathrm{GHz}$ and that of the $\mathrm{TE}_{4,3}$ mode is about $203 \mathrm{GHz}$. Moreover, several SH modes are distributed within a narrow region. The condition for the interaction between the same cyclotron harmonic modes is given by

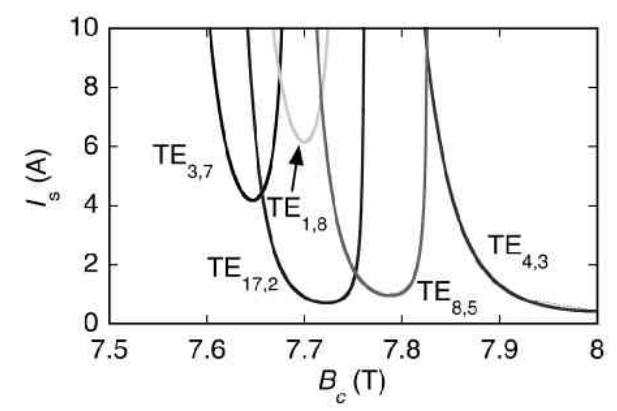

FIG. 1 (color online). Start oscillation currents $I_{s}$ plotted as functions of the cavity field for $V_{k}=60 \mathrm{kV}, R_{b}=1.97 \mathrm{~mm}$, and $\alpha=1.3$. Labels near each curve indicate the oscillation mode. For each mode, $I_{s}$ of either corotating or counterrotating mode with lower current is plotted to avoid unnecessary complication. 


$$
\left|f_{1}-f_{2}\right| \leq \Delta f_{C R}
$$

Here $f_{1}$ and $f_{2}$ are the frequencies of the two modes [2]. The cyclotron resonance band $\Delta f_{C R}$ should be narrower than $1 / T$ with $T=L / \nu_{\|}$being the transit time of an electron with the parallel electron velocity $\nu_{\|}$traveling through the cavity length $L$. In the present study, $\Delta f_{C R} \sim$ $3 \mathrm{GHz}$ and the relation $\left|f_{1}-f_{2}\right| \leq \Delta f_{C R}$ holds between neighboring $\mathrm{SH}$ modes [17]. Indeed, the simultaneous oscillations of two SH modes were observed. This is considered as the nonsynchronous mode interaction [2].

Interaction between the operating $\mathrm{SH}$ mode and the parasitic $\mathrm{FH}$ mode is much more important. In this case, Eq. (1) should read $\left|f_{1}-2 f_{2}\right| \leq \Delta f_{C R}$, where $f_{1}$ and $f_{2}$ are the frequencies of the $\mathrm{SH}$ mode and the $\mathrm{FH}$ mode, respectively. Since $\Delta f_{C R} \leq 1 / T \approx 5 \mathrm{GHz}$ [17], the inequality $\left|f_{1}-2 f_{2}\right| \leq \Delta f_{C R}$ does not hold between the $\mathrm{TE}_{17,2}$ and $\mathrm{TE}_{4,3}$ modes and mode interaction would appear to be difficult. However, two-mode interaction was observed. This interaction is considered as the nonlinear excitation of the $\mathrm{TE}_{4,3}$ mode in the hard self-excitation regime with assistance of the $\mathrm{SH} \mathrm{TE}_{17,2}$ mode in the soft self-excitation regime.

The SH gyrotron used for this study has been developed for application to collective Thomson scattering diagnostics in magnetic fusion devices, which need a $100-\mathrm{kW}$ class gyrotron [18]. Gyrotrons developed for electron heating have been used in several devices [19-21]. The electromagnetic waves with frequencies around $100 \mathrm{GHz}$ suffer from strong refraction and/or absorption in plasma. High-level electron cyclotron emissions are a source of much noise. A sub-terahertz gyrotron will resolve these problems [22,23].

The radius of the cavity of our sub-terahertz gyrotron is $2.99 \mathrm{~mm}$, and the length of the straight section is $12 \mathrm{~mm}$. The cavity has a down taper on the electron-gun side and an up taper connected to a vacuum window via a straight cylindrical waveguide. The maximum beam voltage is $65 \mathrm{kV}$ and the maximum beam current is larger than $10 \mathrm{~A}$. The gyrotron was operated in pulse mode, the pulse length being several microseconds with a repetition rate of less than $10 \mathrm{~Hz}$. See Ref. [17] for design details.

Figure 2 shows the dependence of the oscillation intensity on the magnetic field $B_{c}$ within the cavity. Plots are given for the beam voltage $V_{k}$ of $50 \mathrm{kV}$ [Fig. 2(a)] and $60 \mathrm{kV}$ [Fig. 2(b)]; the beam current $I_{b}$ was $10 \mathrm{~A}$ for both. A pyroelectric detector was located at a distance from one end of a circular waveguide connected to the vacuum window. Dashed and solid lines represent the detector signals without and with a high-pass filter with the cutoff frequency of $303 \mathrm{GHz}$, respectively. The wave forms of the two signals up to $7.55 \mathrm{~T}$ in Fig. 2(a) and up to $7.6 \mathrm{~T}$ in Fig. 2(b) almost coincide with each other, indicating that almost all the signal in this region is attributed to an $\mathrm{SH}$ oscillation. It is also noticeable that the FH mode region connects with the $\mathrm{SH}$ mode region with no gap.

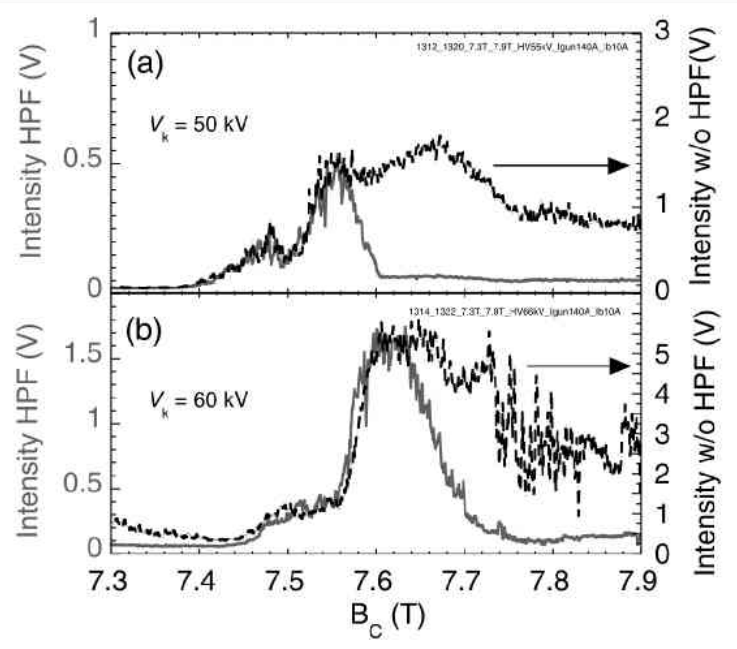

FIG. 2 (color online). Oscillation intensities for (a) $V_{k}=50 \mathrm{kV}$ and (b) $V_{k}=60 \mathrm{kV}$ are plotted as functions of the cavity field. Solid lines represent signals with the highpass filter; dashed lines are those without the high-pass filter.

Figure 3 displays the transmission signal from a Fabry-Perot interferometer as a function of the distance between reflecting meshes. The beam voltage was $60 \mathrm{kV}$. Figure 3(a) is the transmission signal at 7.6 T; Fig. 3(b) is for $7.68 \mathrm{~T}$. Figure 3(a) shows a single-mode $\mathrm{SH}$ oscillation, identified as the $\mathrm{TE}_{17,2}$ mode from accurate frequency measurements using a heterodyne receiver system [17]. At 7.68 T, the SH oscillation power decreased [Fig. 2(b)] and the FH mode oscillation increased. Figure 3(b) includes two peak trains with different intervals. The train with lower peak values is attributed to the $\mathrm{TE}_{17,2}$ mode; the higher-peak train has a longer interval about twice as long as that of the $\mathrm{TE}_{17,2}$ mode. Frequency analysis identifies it

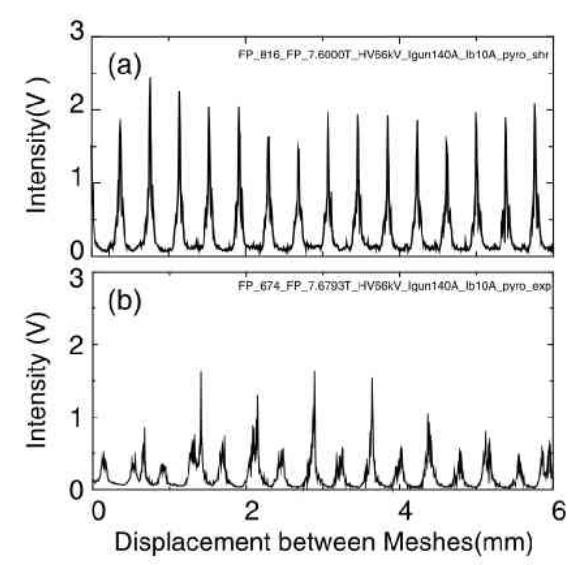

FIG. 3. Transmission signals through a Fabry-Perot interferometer plotted as functions of distance between the reflecting meshes: (a) $B_{c}=7.6 \mathrm{~T}$ and (b) $B_{c}=7.68 \mathrm{~T}$. The beam voltage $V_{k}$ was $60 \mathrm{kV}$; the beam current $I_{b}$ was $10 \mathrm{~A}$. 

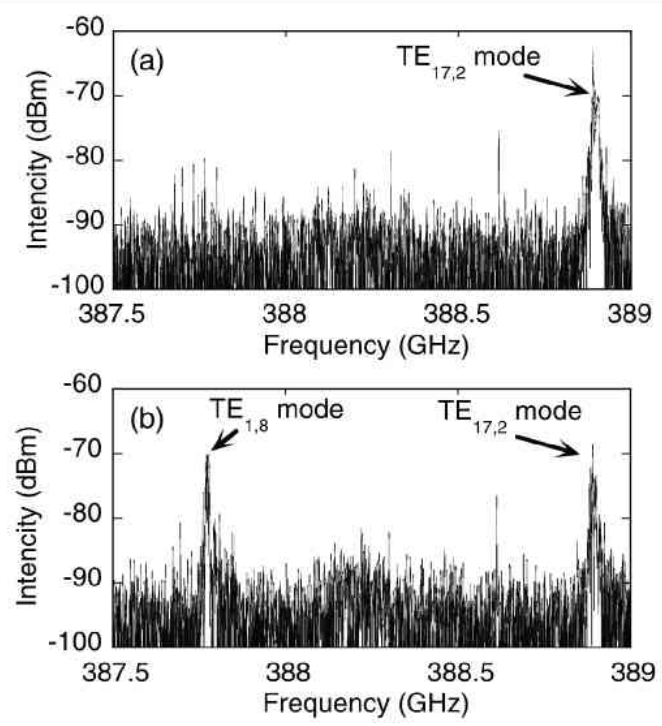

FIG. 4. Frequency spectra for the intermediate frequency signal from the harmonic mixer of the heterodyne receiver system: (a) $R_{b}=1.97 \mathrm{~mm}$ and (b) $R_{b}=2.05 \mathrm{~mm}$. The abscissa is converted to a rf scale.

as the $\mathrm{TE}_{4,3}$ mode. Simultaneous oscillation of the $\mathrm{SH}$ and FH modes was observed.

The second observation concerns the interaction among $\mathrm{SH}$ modes. Figure 4 plots frequency spectra of the intermediate frequency signal from a harmonic mixer of the heterodyne receiver. The cavity field was $7.6 \mathrm{~T}$ corresponding to the peak of the SH mode signal in Fig. 2(b). Only one peak corresponding to the $\mathrm{TE}_{17,2}$ mode is distinguished in Fig. 4(a) for the same condition as the highest power oscillation [17]. The electron beam radius $R_{b}$ was set at $1.97 \mathrm{~mm}$ where the coupling coefficient of the competing $\mathrm{TE}_{1.8}$ mode is almost zero. A small variation of $R_{b}$ resulted in a new peak as shown in Fig. 4(b). The frequency interval between the two peaks is just equal to the difference between the frequencies $388.89 \mathrm{GHz}$ of the $\mathrm{TE}_{17,2}$ mode and $387.77 \mathrm{GHz}$ of the $\mathrm{TE}_{1,8}$ mode. Simultaneous oscillation of two SH modes was observed.

We discuss next the dynamic behavior of the observed mode interactions. Figure 5 represents mode interaction calculations $[17,24]$ for $R_{b}=1.97 \mathrm{~mm}$. The calculation producing Fig. 5(a) takes into account four $\mathrm{SH}$ modes, $\mathrm{TE}_{3,7}, \mathrm{TE}_{1,8}, \mathrm{TE}_{17,2}$, and $\mathrm{TE}_{8,5}$, whereas for Fig. 5(b) these modes are not included. The range of $B_{c}$ for the $\mathrm{TE}_{4,3}$ mode oscillation substantially extends to the low field side in Fig. 5(a) as compared with the range in Fig. 5(b). There is no oscillation gap around $7.7 \mathrm{~T}$ in Fig. 5(a). The $B_{c}$ region of the $\mathrm{TE}_{17.2}$ mode oscillation agrees well with that in Fig. 2(b). Temporal evolution of the relevant modes calculated at $B_{c}=7.69 \mathrm{~T}$ under fixed voltage and current is plotted in Fig. 6. The $\mathrm{TE}_{17,2}$ mode is first excited, and then the $\mathrm{TE}_{4,3}$ mode grows. A steady state of the two-mode
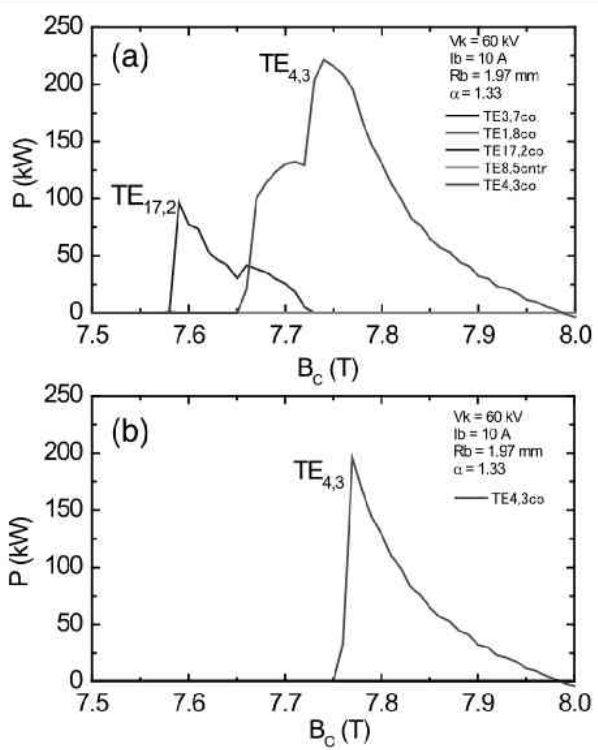

FIG. 5 (color online). Mode interaction calculations: (a) the relevant four SH modes are taken into account; (b) only the FH $\mathrm{TE}_{4,3}$ mode is included in the calculation $\left(V_{k}=60 \mathrm{kV}\right.$, $R_{b}=1.97 \mathrm{~mm}, \alpha=1.33$ ).

oscillation is established within tens of nanoseconds, that being much shorter than the pulse width. Therefore, the experimental observation shown in Fig. 3(b) corresponds to the steady state.

Mode interaction in gyrotrons has been discussed from various angles. Hard self-excitation of an $\mathrm{SH}$ mode with the help of a parasitic FH mode was theoretically found [25]. However, the SH TE $\mathrm{T}_{17,2}$ mode is self-excited, and moreover, the condition requiring instant rise of beam voltage does not hold in the present experiment.

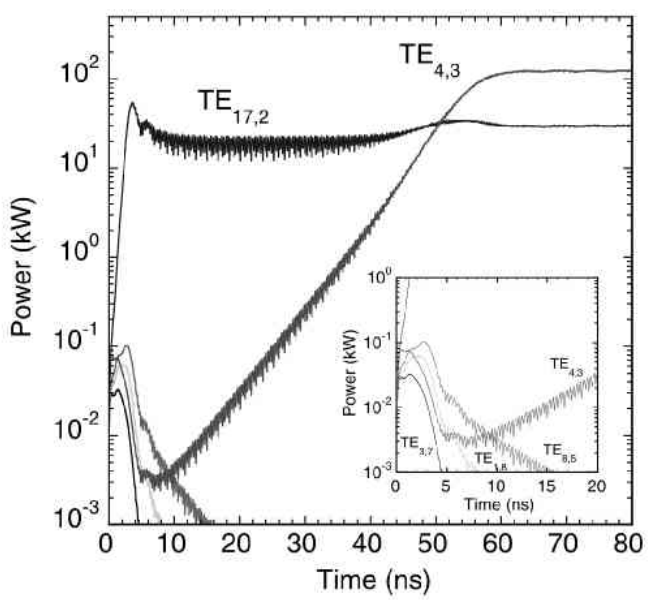

FIG. 6 (color online). Temporal evolution of the $\mathrm{TE}_{4,3}$ mode and the $\mathrm{TE}_{17.2}$ mode at $B_{c}=7.69 \mathrm{~T}$ is plotted on the mode interaction calculation $\left(V_{k}=60 \mathrm{kV}, R_{b}=1.97 \mathrm{~mm}\right.$, $\alpha=1.33$ ). The inset displays the start-up phase. 


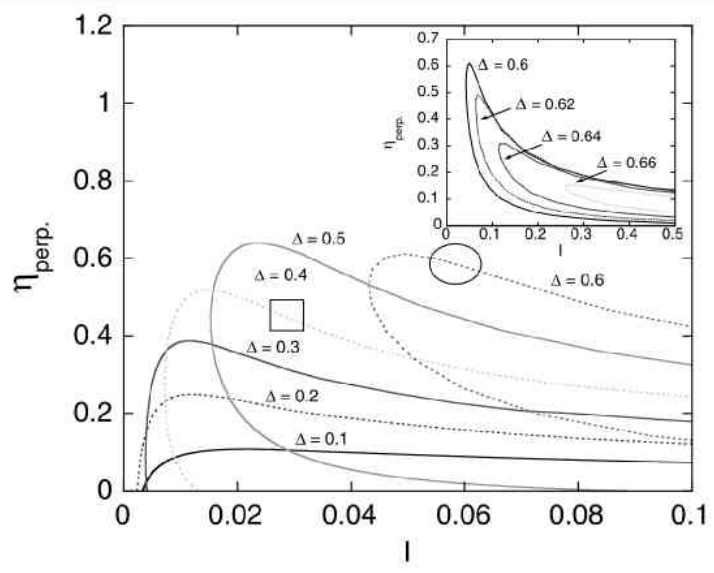

FIG. 7 (color online). Perpendicular efficiency is plotted as a function of the normalized current for different values of the frequency detuning parameter $\Delta$. The inset gives the same calculation for various $\Delta>0.6$.

Interaction between arbitrary cyclotron harmonics was studied self-consistently [26]. This analysis predicts hard self-excitation of a parasitic FH mode over a wide range of $\Delta$ with the presence of an SH mode.

The present study has shown the nonlinear hard selfexcitation of the parasitic FH mode that has been theoretically predicted $[1,2,10]$. We enumerate arguments to verify the nonlinear excitation of the $\mathrm{TE}_{4,3}$ mode in the hard self- excitation regime.

First, as shown in Fig. 5(b), oscillation of the $\mathrm{TE}_{4,3}$ mode alone is not possible for $B_{c}$ lower than $7.75 \mathrm{~T}$ and assistance from the $\mathrm{TE}_{17,2}$ mode is necessary. Moreover, the highest power is obtained at around 7.75 $\mathrm{T}$. These indicate that the $\mathrm{TE}_{4,3}$ mode is in the hard self-excitation regime for $B_{c}$ lower than $7.75 \mathrm{~T}$.

Second, the frequency detuning parameter $\Delta$ for the $\mathrm{FH}$ $\mathrm{TE}_{4,3}$ mode is about 0.6 at $7.75 \mathrm{~T}$ and larger than 0.8 at 7.7 T. With $\Delta$ as a parameter, the perpendicular efficiency $\eta_{\perp}$ of the $\mathrm{TE}_{4,3}$ mode is plotted as a function of the normalized current $I$ (Fig. 7). The definitions of $\eta_{\perp}$ and $I$ are given in Ref. [11]. The normalized interaction length $\mu$ is set at 13 , corresponding to the present experiment; the circle in Fig. 7 indicates its parameter region. The value of $I$ is smaller than the start oscillation current at which $\eta_{\perp}=0$. This region is then in the hard self-excitation regime. The square in Fig. 7 represents the parameter region of the previous mode cooperation experiment $[9,10]$ and is located in the soft self-excitation regime. The inset in Fig. 7 depicts $\eta_{\perp}$ for $\Delta>0.6$. The value of $\eta_{\perp}$ rapidly decreases with $\Delta$ and goes down to almost zero for $\Delta=0.7$. This calculation does not include mode interaction. Therefore, the oscillation of the $\mathrm{TE}_{4,3}$ mode at $B_{c}<$ 7.7 $\mathrm{T}$ is due to the nonlinear excitation. A mode-interaction calculation also confirms the nonlinear excitation of the $\mathrm{TE}_{4,3}$ mode for $V_{k}=50 \mathrm{kV}$ as shown in Fig. 2(a).
Third, the inset in Fig. 6 clearly indicates the dynamic process of the mode interaction. The $\mathrm{TE}_{17,2}$ mode suppresses the $\mathrm{TE}_{4,3}$ mode at the very early stage. The $\mathrm{TE}_{4,3}$ mode starts to increase after about $5 \mathrm{~ns}$ and grows under the earlier saturated $\mathrm{TE}_{17,2}$ mode. This time scale is consistent with the cavity filling time $\tau_{d} \sim Q / \omega$, where $Q$ is the total $Q$ value as a result of the diffractive $Q$ value and the Ohmic $Q$ value. The total $Q$ values of the $\mathrm{SH}$ modes are about 5300 except for the $\mathrm{TE}_{17,2}$ mode, which has a somewhat lower value of about 4300 because of a rather lower Ohmic $Q$ value. The total $Q$ value of the $\mathrm{TE}_{4,3}$ mode is about 1700 . Therefore, $\tau_{d}$ for the SH modes are around $2 \mathrm{~ns}$ and that for $\mathrm{TE}_{4,3}$ mode is about $1 \mathrm{~ns}$. Preliminary excitation of the $\mathrm{TE}_{17,2}$ mode in the soft self-excitation regime enables the excitation of the $\mathrm{TE}_{4,3}$ mode in the hard selfexcitation regime. This is consistent with the picture of the nonlinear hard self-excitation. The decay of $\mathrm{SH}$ modes other than the $\mathrm{TE}_{17,2}$ mode in the start-up phase suggests parametric interaction between the $\mathrm{SH}$ modes and the $\mathrm{TE}_{4,3}$ mode. Three runs [(1) only $\mathrm{TE}_{17,2}$ and $\mathrm{TE}_{4,3}$; (2) $\mathrm{TE}_{17,2}, \mathrm{TE}_{8,5}$, and the $\mathrm{TE}_{4,3}$; and (3) $\mathrm{TE}_{17,2}, \mathrm{TE}_{8,5}$, $\mathrm{TE}_{1,8}$, and $\mathrm{TE}_{4,3}$ modes] were carried out. The rotation directions of these modes were prescribed depending on the magnitude of the coupling coefficient at $R_{b}=$ $1.97 \mathrm{~mm}$. No distinguishable difference on the excitation of the $\mathrm{TE}_{4,3}$ mode was observed. The predominantly large coupling coefficient of the $\mathrm{TE}_{17,2}$ mode determines the dynamic interaction with the $\mathrm{TE}_{4,3}$ mode.

Fourth, we consider two normalized parameters $\theta_{s}=$ $\Delta_{s} \mu_{s}$ and $k=\theta_{s^{\prime}} / \theta_{s}$ related with the analyses given in Ref. [1]. Here, the subscripts $s$ and $s^{\prime}$ denote the $\mathrm{TE}_{4.3}$ mode and the $\mathrm{TE}_{17,2}$ mode, respectively. By substituting corresponding values $\left(\Delta_{s} \sim 0.6, \Delta_{s^{\prime}} \sim 0.2, \mu_{s} \sim 13\right.$, and $\left.\mu_{s^{\prime}} \sim 23\right), \theta_{s}$ and $k$ are evaluated as about 8 and 0.6, respectively. These values correspond to the nonlinear hard self-excitation regime in Fig. 3(b) of Ref. [1], where the nonlinear interaction coefficient $\gamma_{s s^{\prime}}$ becomes negative. Another parameter is $\tilde{\Delta}=\Delta_{s^{\prime}}-\Delta_{s}$. For the present case, $\tilde{\Delta}$ is about 0.4 , which also corresponds to expansion of the field in which a parasitic mode can be nonlinearly hard self-excited as shown in Fig. 6 of Ref. [1]. This is consistent with the low-field side extension of the $B_{c}$ range for $\mathrm{TE}_{4,3}$ mode oscillation [Fig. 2(b)].

The $\mathrm{TE}_{17,2}$ mode does not oscillate at $7.75 \mathrm{~T}$ in the steady state. Temporal evolution calculation shows that the $\mathrm{TE}_{17,2}$ mode is first excited at the start-up stage and then decays to zero. The $\mathrm{TE}_{4,3}$ mode grows with assistance from the $\mathrm{TE}_{17,2}$ mode, and once it has grown, its power level remains constant. Both cases of stable two-mode oscillation and oscillation of the $\mathrm{TE}_{4,3}$ mode only are possible depending on $B_{c}$. This indicates strong coupling between the hard and soft self-excited modes [1].

The interaction between the $\mathrm{TE}_{17,2}$ and $\mathrm{TE}_{1,8}$ modes is a good evidence of the nonsynchronous interaction [2]. A change of $R_{b}$ results in the transition from strong coupling, 
for which only one-mode oscillation is stable, to weak coupling for which two modes coexist.

In summary, both experimental and theoretical analyses verify the interaction between $\mathrm{FH}$ and $\mathrm{SH}$ modes as the observation of the nonlinear excitation of the $\mathrm{FH}$ mode in the hard self-excitation regime. These modes are strongly coupled and stable two-mode oscillation as well as oscillation of the FH mode only were observed.

The present study was supported by programs of the Grants-in-Aid for Scientific Research of the Japan Society for the Promotion of Science.

[1] G. S. Nusinovich, Int. J. Electron. 51, 457 (1981).

[2] G. S. Nusinovich, IEEE Trans. Plasma Sci. 27, 313 (1999) and papers cited therein.

[3] V.L. Bratman et al., IEEE Trans. Plasma Sci. 37, 36 (2009).

[4] T. Idehara and S. Sabchevski, J. Infrared Millim. Terahz. Waves 33, 667 (2012).

[5] V.L. Bratman, Yu. Kalynov, and V. Manuilov, Phys. Rev. Lett. 102, 245101 (2009).

[6] S. H. Kao, C. Chiu, K. Pao, and K. Chu, Phys. Rev. Lett. 107, 135101 (2011).

[7] S. H. Kao, C.C. Chiu, and K. R. Chu, Phys. Plasmas 19, 023112 (2012).

[8] K.E. Kreischer, R.J. Temkin, H. R. Fetterman, and W. J. Mulligan, IEEE Trans. Microwave Theory Tech. 32, 481 (1984).

[9] T. Idehara and Y. Shimizu, Phys. Plasmas 1, 3145 (1994).
[10] T. Idehara, S. Mitsudo, M. Pereyaslavets, Y. Shimizu, and I. Ogawa, Int. J. Infrared Millim. Waves 20, 1249 (1999).

[11] B. G. Danly and R. J. Temkin, Phys. Fluids 29, 561 (1986).

[12] G. S. Nusinovich, Introduction to the Physics of Gyrotrons (Johns Hopkins University, Baltimore, 2004).

[13] V.E. Zapevalov, S. A. Malygin, and Sh.E. Tsimring, Radiophys. Quantum Electron. 36, 346 (1993).

[14] S. Spira-Hakkarainen, K. E. Kreischer, and R. J. Temkin, IEEE Trans. Plasma Sci. 18, 334 (1990).

[15] T. Notake, T. Saito, Y. Tatematsu, A. Fujii, S. Ogasawara, La Agusu, I. Ogawa, and T. Idehara, Phys. Rev. Lett. 103, 225002 (2009).

[16] T. Saito, S. Ogasawara, N. Yamada, S. Ikeuchi, Y. Tatematsu, R. Ikeda, I. Ogawa, and V.N. Manuilov, Plasma Fusion Res. 7, 1206003 (2012).

[17] T. Saito et al., Phys. Plasmas 19, 063106 (2012).

[18] P. Woskoboinikov, Rev. Sci. Instrum. 57, 2113 (1986).

[19] W. V. Suvorov et al., Plasma Phys. Controlled Fusion 37, 1207 (1995).

[20] H. Bindslev et al., Plasma Fusion Res. 2, S1023 (2007).

[21] M. Nishiura et al., Plasma Fusion Res. 6, 2402068 (2011).

[22] T. Notake et al., Rev. Sci. Instrum. 79, 10E732 (2008).

[23] T. Saito et al., J. Phys. Conf. Ser. 227, 012013 (2010).

[24] Y. Tatematsu, Y. Yamaguchi, T. Idehara, T. Ozeki, R. Ikeda, T. Kanemaki, I. Ogawa, and T. Saito, J. Infrared Millim. Terahz. Waves 33, 292 (2012).

[25] G. S. Saraph, T. M. Antonsen, G. S. Nusinovich, and B. Levush, Phys. Fluids B 5, 4473 (1993).

[26] O. Dumbrajs, G. S. Nusinovich, and A. B. Pavelyev, IEEE Trans. Plasma Sci. 18, 301 (1990). 